\title{
Experimental test for extra dimensions in Kaluza-Klein gravity
}

\author{
V. Dzhunushaliev * \\ Universität Potsdam, Institute für Mathematik, 14469, Potsdam, Germany \\ and Theor. Phys. Dept. KSNU, 720024, Bishkek, Kyrgyzstan \\ D. Singleton ${ }^{\dagger}$ \\ Dept. of Phys. CSU Fresno, 2345 East San Ramon Ave. M/S 37 Fresno, CA 93740-8031, USA
}

5 D Kaluza-Klein gravity has several nonasymptotically flat solutions which generally, possessed both electric and magnetic charges. In this paper we suggest that these solutions can act as quantum virtual handles (wormholes) in spacetime foam models. By applying a sufficently large, external electric and/or magnetic field it may be possible to "inflate" these solutions from a quantum to a classical state. This effect would lead to a possible experimental signal for higher dimensions in multidimensional gravity.

\section{INTRODUCTION}

Most modern theories which attempt to unify gravity with the Standard Model gauge theory have extra dimensions. These extra dimensions makes it possible to geometrize the gauge fields (gauge bosons) according to the following theorem [1]:

Let $G$ be the group fibre of the principal bundle. Then there is a one-to-one correspondence between the $G$-invariant metrics

$$
d s^{2}=h^{2}\left(x^{\mu}\right)\left(\sigma^{a}+A_{\mu}^{a} d x^{\mu}\right)^{2}+g_{\mu \nu} d x^{\mu} d x^{\nu}
$$

on the total space $\mathcal{X}$ and the triples $\left(g_{\mu \nu}, A_{\mu}^{a}, h\right)$. Here $g_{\mu \nu}$ is Einstein's pseudo - Riemannian metric on the base; $A_{\mu}^{a}$ are the gauge fields of the group $G$ ( the nondiagonal components of the multidimensional metric); $h \gamma_{a b}$ is the symmetric metric on the fibre.

The off-diagonal components of multidimensional (MD) metric act as Yang-Mills fields. One distinction between such MD theories and 4D theories with Yang-Mills fields is that the MD theories have a scalar field connected with the extra dimension(s). This scalar field describes the linear size of the extra dimensions or equivalently the volume of gauge group. Thus one possible experimental test for any MD gravity theory is the observation of effects

*E-Mail Address : dzhun@freenet.bishkek.su

${ }^{\dagger}$ E-Mail Address : das3y@maxwell.phys.csufresno.edu 
arising from this scalar field. For example, it is possible to show that in 5D Kaluza - Klein theory the presence of variations of the $5^{\text {th }}$ coordinate leads to changes in the ratio of the electrical charge to the mass of an elementary particle. This effect is very small since no experiment has found such a change.

In this paper we offer a new possible experimental signal for probing the extra dimensions of MD gravity based on the existence of a certain type of spherically symmetric nonasymptotically flat solutions [2].

\section{WORMHOLE AND FLUX TUBE SOLUTIONS IN 5D GRAVITY.}

Before discussing the 5D solutions we will briefly recall a couple of spherically symmetric 4D electrogravity solutions which will be used for comparison with the 5D solutions. First there is the well known, asymptotically flat generalized Reissner-Nordström solution which gives the gravitational and electromagnetic fields for a point mass with both electric and magnetic charges (the time-time component of the metric has the form $g_{t t}=\left(1-\frac{m}{r}+\frac{q^{2}+Q^{2}}{r^{2}}\right)$ where $m$ is the mass and $q, Q$ are the electric and magnetic charges respectively). Second, there is the nonasymptotically flat, spherically symmetric Levi-Civita flux tube solution [3] with the metric

$$
\begin{aligned}
& d s^{2}=a^{2}\left(\cosh ^{2} \zeta d t^{2}-d \zeta^{2}-d \theta^{2}-\sin ^{2} \theta d \varphi^{2}\right), \\
& F_{01}=\rho^{1 / 2} \cos \alpha, \quad F_{23}=\rho^{1 / 2} \sin \alpha,
\end{aligned}
$$

where $G^{1 / 2} a \rho^{1 / 2}=1 ; \alpha$ is an arbitrary constant angle; $a$ and $\rho$ are constants defined by Eq. (2) - (3) ; $G$ is Newton's constant ( $c=1$, is the speed of light); $F_{\mu \nu}$ is the electromagnetic field tensor. Both the generalized Reissner-Nordström solution and the Levi-Civita flux tube solution place no restrictions on the relative values of the electric and magnetic charges.

In 5D Kaluza - Klein theory there are intriguing wormhole (WH) and flux tube solutions [2] [4]. Here we give a brief summary of these solutions. The general form of the metric is:

$$
\begin{aligned}
d s^{2} & =e^{2 \nu(r)} d t^{2}-r_{0}^{2} e^{2 \psi(r)-2 \nu(r)}[d \chi+\omega(r) d t+n \cos \theta d \varphi]^{2} \\
& -d r^{2}-a(r)\left(d \theta^{2}+\sin ^{2} \theta d \varphi^{2}\right)
\end{aligned}
$$

where $\chi$ is the $5^{\text {th }}$ coordinate; $\omega=A_{t}$ and $n \cos \theta=A_{\phi}$ are the $4 \mathrm{D}$ electromagnetic potentials; $n$ is an integer; $r, \theta, \varphi$ are "polar" coordinates. The $5 \mathrm{D}$ spacetime is the total space of the $\mathrm{U}(1)$ principal bundle, where the fibre is the $\mathrm{U}(1)$ gauge group and the base is ordinary $4 \mathrm{D}$ spacetime.

A detailed analytical and numerical investigation of the metric in Eq. (4) gives the following spacetime configurations, whose global structure depends on the relationship between the electric and magnetic fields [四]:

1. $0 \leq H_{K K}<E_{K K}$. The corresponding solution is $\boldsymbol{a} \boldsymbol{W H}$-like object located between two surfaces at $\pm r_{0}$ where the reduction from $5 \mathrm{D}$ to $4 \mathrm{D}$ spacetime breaks down [5]. 
The cross-sectional size of this solution (given by $a(r)$ ) increases as $r$ goes from 0 to $\pm r_{0}$. The throat between the $\pm r_{0}$ surfaces is filled with electric and/or magnetic flux. As the strength of the magnetic field increases the longitudinal distance between the surfaces at $\pm r_{0}$ increases. This can be seen diagrammatically from the first two pictures in Fig.1.

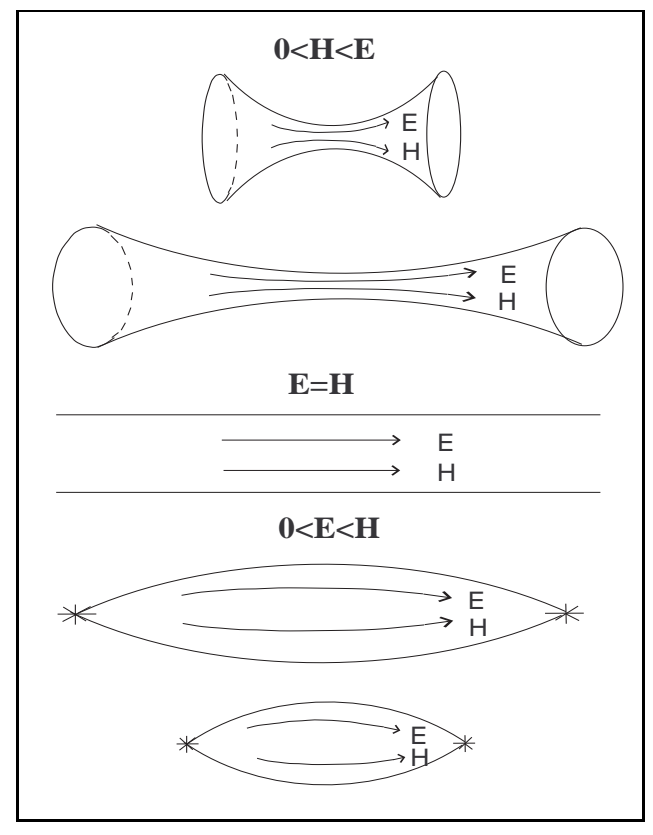

FIG. 1. The evolution from WH-like solution to finite flux tube solution

2. $H_{K K}=E_{K K}$. In this case the solution is an infinite flux tube filled with constant electrical and magnetic fields, with the charges disposed at $\pm \infty$. The cross-sectional size of this solution is constant $(a=$ const.). In Refs. [4] [8] an exact, analytical form of this solution was given in terms of hyperbolic functions. This solution if almost identical to the $4 \mathrm{D}$ Levi-Civita flux tube solution except the strength of the magnetic and electric fields are equal, while in the Levi-Civita solution the two fields can take on any relative value with respect to one another. The restriction that the electric charge equals the magnetic charge is reminiscent of other higher dimensional soliton solutions. In Ref. [6] non-Abelian, Kaluza-Klein dyon solutions were found which obeyed the same restriction that the "electric" charge equal the "magnetic" charge. The present flux tube solution can be viewed as two connected or bound Kaluza-Klein dyons. The form of this infinite flux tube configuration also has similarities to the Anti-de Sitter (AdS) "throat region" that one finds by stacking a large number of D3-branes [7]. Both the spacetime around the D3-branes and the electric/magnetic flux tube have indefinitely long cylindrical "throats" which can be thought of as ending either on the horizon of a black hole (for the D3-branes solution), or on an electric/magnetic charged object 
(for the flux tube solution).

3. $0<E_{K K} \leq H_{K K}$. In this case we have $\boldsymbol{a}$ finite flux tube between two $(+)$ and (-) magnetic and/or electric charges, which are located at $\pm r_{0}$. The longitudinal size of this flux tube is finite, but now the cross sectional size decreases as $r \rightarrow r_{0}$. At $r= \pm r_{0}$ this solution has real singularities which we interpret as the locations of the magnetic and/or and electric charges. The behavior of this flux tube solution as $E_{K K}$ decreases can be seen diagrammatically from the last two pictures in Fig. 1

\section{THE BASIC IDEA}

In Ref. [9] the idea was advanced that a piecewise compactification mechanism can exist in Nature. Piecewise compactification implies that some parts of the Universe are regions where one has full MD gravity (5D in our case), while other parts of the Universe are ordinary 4D regions where gravity does not act on the extra dimensions. For this mechanism to be viable it is necessary that on the boundary between these regions a quantum splitting off of the $5^{\text {th }}$ dimension occurs. In regions where gravity propagates in all the dimensions the Universe will appear as a true 5D spacetime. I In the regions where gravity does not propagate into the extra dimension one has ordinary 4D spacetime plus the gauge fields of the fibre. [2. The boundary between these regions should be Lorentz invariant surfaces [? An example of such a construction is the composite WH of Ref. [8] which consists of two 4D Reissner - Nordström black holes attached to either end of a 5D WH solution (see Fig.2).

\footnotetext{
${ }^{1}$ in this case the fifteen $5 \mathrm{D}$ Einstein vacuum equations $=4 \mathrm{D}$ gravity + Maxwell electrodynamic + scalar field.

${ }^{2}$ in this case there are fourteen $5 \mathrm{D}$ Einstein vacuum equations $=4 \mathrm{D}$ gravity + Maxwell electrodynamic, where $G_{55}=$ scalar field does not vary.

${ }^{3}$ for the $4 \mathrm{D}$ observer at infinity it will appear as an event horizon.
} 


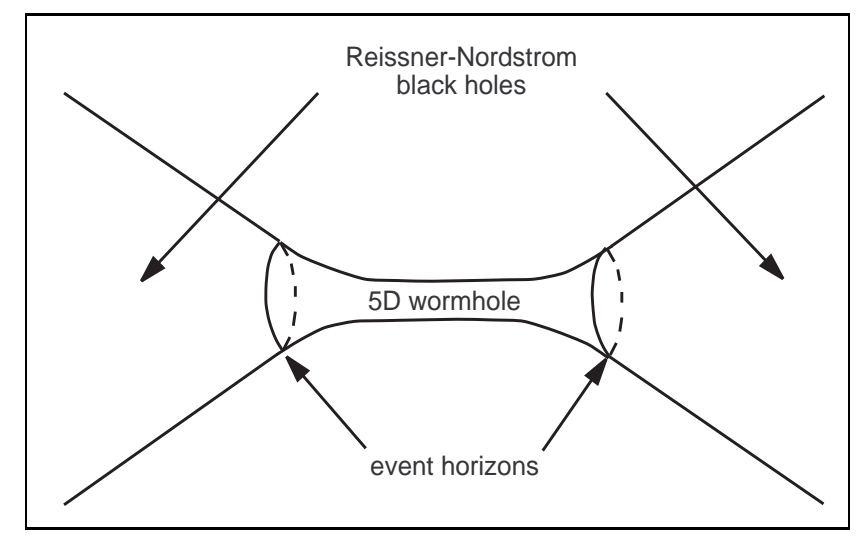

FIG. 2. The forces acting on 2 ends of WH

The proposed experimental signal for the extra dimensions in MD gravity relies on these postulated composite WH structures. The basic idea is the following: Composite WHs can act as a quantum handles (quantum WHs) in the spacetime foam. These quantum structures can be "blown up" or "inflated" from a quantum state to a classical state by embedding it in parallel $E$ and $H$ fields with $E>H$. These quantum handles are taken as quantum fluctuations in the spacetime foam which the externally imposed $E$ and $H$ fields can then promote to classical states with some probability. This process is envisioned as taking place inside a solenoid which has an additional electric field, $E$, parallel to magnetic field $H$.

The above process has some similarity to the pair production of electric or magnetic charged black holes in an external electric or magnetic field [11] 12]. In Ref. [12 it was shown that despite the fact that the Maxwell action $\left(F_{\mu \nu} F^{\mu \nu}=\mathbf{H}^{2}-\mathbf{E}^{2}\right)$ changes sign under a dual transformation of $\mathbf{H}$ and $\mathbf{E}$ that the pair production of electric black holes and magnetic black holes are identical and suppressed. In the next section we consider an approximate flux tube solution which has $\mathbf{E} \approx \mathbf{H}$ and therefore has a Maxwell action which is approximately zero.

\section{A MORE DETAILED DESCRIPTION}

The first three pictures in Fig. 1 represent solutions where the charges are unconfined and separated by some finite, longitudinal distance. For an external observer these composite WHs will appear as two oppositely charged electric/magnetic objects, with the charges located on the surfaces where the 4D and 5D spacetimes are matched. Since one would like these electric/magnetic charged objects to be well separated, we will consider the case $E \approx H(E>H)$. (This leads to the Maxwell action, $F^{2}=H^{2}-E^{2}$, being approximately zero). Under these condition the solution to Einstein's MD vacuum equations for the metric ansatz given in Eq. (四) is [4] 10] 


$$
\begin{array}{r}
q \approx Q, \\
a \approx \frac{q^{2}}{2}=\text { const }, \\
e^{\psi} \approx e^{\nu} \approx \cosh \left(\frac{r \sqrt{2}}{q}\right), \\
\omega \approx \frac{\sqrt{2}}{r_{0}} \sinh \left(\frac{r \sqrt{2}}{q}\right)
\end{array}
$$

here $q$ is the electrical charge and $Q$ the magnetic charge. Both Kaluza - Klein fields are:

$$
E \approx H \approx \frac{q}{a} \approx \frac{2}{q} \approx \sqrt{\frac{2}{a}}
$$

The cross sectional size of the WH is proportional to $q^{2}$. According our scenario the external, parallel electric and magnetic fields should fill the virtual WH. Changing Eqs. (5)-(8) into cgs units the electric and magnetic fields necessary for forming a composite WH with a cross sectional size $a$ is

$$
E \approx H \approx \frac{c^{2}}{\sqrt{G}} \sqrt{\frac{2}{a}}
$$

From Eq. (10) it can be seen that the larger the cross sectional size, $a$, of the WH the smaller the $E$ and $H$ fields. However, in order for the charged surfaces of the WH to appear as well separated electric/magnetic charged objects we need to require that the longitudinal distance, $l$, between these surfaces be much larger than the cross sectional size of the WH, $l \gg \sqrt{a}$. Also in order to be able to separate the two ends of the WH as distinct electric/magnetic charged objects one needs the external force to be much larger than the interaction force between the oppositely charged ends. This leads to the following condition which is illustrated in Fig.3.

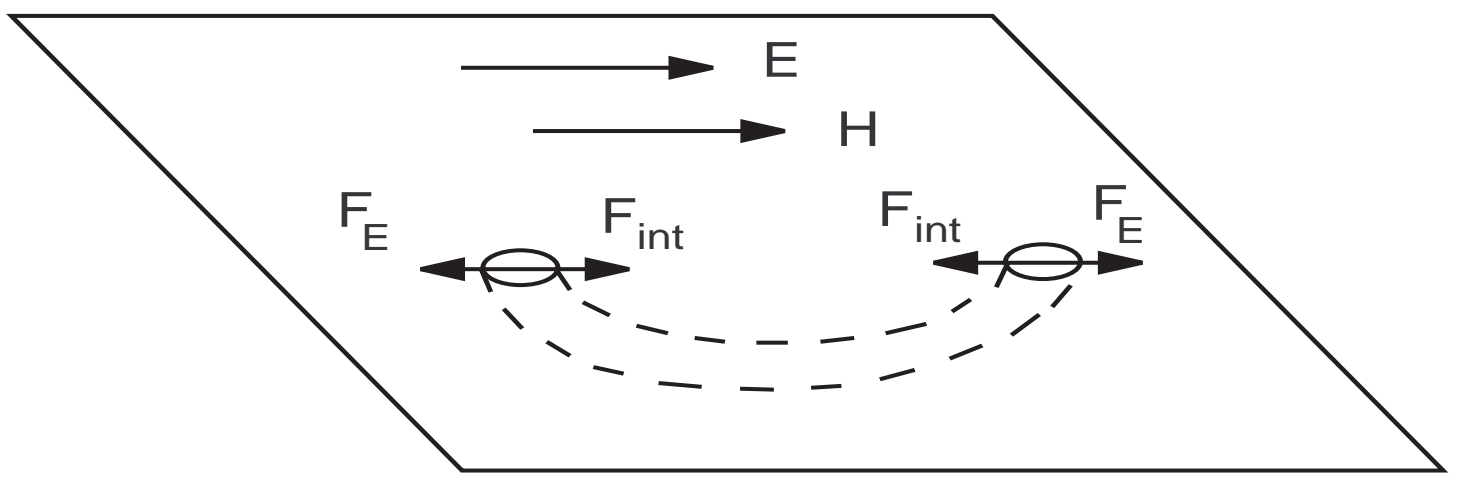

FIG. 3. The forces acting on 2 ends of WH 


$$
\begin{aligned}
F_{\text {ext }} & =q E+Q H \gg \frac{q^{2}+Q^{2}}{l^{2}}=F_{\text {int }} \\
& \approx 2 q E \gg \frac{2 q^{2}}{l^{2}} \rightarrow l \gg \sqrt{a}
\end{aligned}
$$

If this condition holds than the oppositely charged ends will move apart. Otherwise the ends will come back together and annihilate back into the spacetime foam.

The average value of $a$ for spacetime foam is given by the Planck size $\sqrt{a} \approx L_{P l} \approx 10^{-33}$ cm. Thus the relevant field $E$ should be $E \approx \sqrt{2 c^{7}} / G \sqrt{\hbar} \approx 3.1 \times 10^{57} \mathrm{~V} / \mathrm{cm}$. This field strength is in the Planck region, and is well beyond experimental capabilities to create. Hence one must consider quantum WHs whose linear size satisfies $\sqrt{a} \gg L_{P l}$. The larger $\sqrt{a}$ the smaller the field strength needed. But such large quantum WHs are most likely very rare. If $f(a)$ is the probability density for the distribution for a WH of cross section $a$ then $f(a) d a$ gives the probability for the appearance of quantum WH with cross section $a$. The bigger $a$ the smaller the probability, $f(a) d a$. Also the larger the value of $E$ and $H$ the smaller is the cross sectional size $a$ of the WH that can be inflated from the spacetime foam. Thus depending on the unknown probability $f(a) d a$ one can set up some spatial region with parallel $E$ and $H$ fields whose magnitudes are as large as technologically feasible, and look for electric/magnetic charged objects whose charges are of similar magnitude. Finally, it has been proposed [13] that the Planck scale may occur at a much lower energy scale $\left(\approx 10^{3} \mathrm{GeV}\right)$ than is normally thought $\left(\approx 1 \times 10^{19} \mathrm{GeV}\right)$ due to the presence of large, extra dimensions. In such a scenario $\sqrt{a} \approx L_{P l} \approx 10^{-18} \mathrm{~cm}$, and the field strength would decrease by fifteen orders of magnitude so that $E \approx 3.1 \times 10^{42}$ from above. This is still beyond experimental capabilities, however now one can consider quantum WHs that are of a smaller size, $a$, as compared to the standard case when the Planck size is $10^{-33} \mathrm{~cm}$. Combining the large extra dimension scenario with the inflation of the electric/magnetic infinite flux tube solution by external fields, tends to increase the probability of observing such an event.

The energy density $u$ of electrical and magnetic fields stored in such an inflated WH is

$$
u=\frac{E^{2}}{8 \pi}+\frac{H^{2}}{8 \pi} \approx \frac{E^{2}}{4 \pi}=\frac{1}{4 \pi} \frac{c^{4}}{G} \frac{2}{a} .
$$

In this case the energy $U$ is

$$
U \approx \pi a l w=\frac{c^{4}}{2 G} l
$$

$U$ increases linearly with $l$ as one would expect for two objects connected by a flux tube. This places a restriction on $l$, since as $l$ increases beyond a certain point the energy will be large enough to favor creating another electric/magnetic charged pair. 


\section{CONCLUSION}

We have presented a possible experimental scheme to test the presence of the higher dimensions in MD gravity through the use of certain WH-like solutions, and an assumption about piecewise compactification on the surface where the reduction from 5D to 4D breaks down.

The difference between the present solutions the 4D Levi-Civita flux tube solution is that the 5D solution requires that the magnitudes of the electric and magnetic charges be of the same magnitude. This restriction on the charges is similar to that for certain non-Abelian, Kaluza-Klein dyon solutions [6]. In the 4D case any relative strength between the charges is allowed. For both the 4D and 5D solutions it can be asked how the Dirac condition between electric/magnetic charges fits into all of this. Recently an investigation into closely related cosmic magnetic flux tube solutions was carried out [14]. It was found that in the context of these GR solutions the Dirac condition is modified so that (magnetic flux) + (dual electric charge) is the quantized object rather than just the magnetic flux.

\section{ACKNOWLEDGMENTS}

VD is supported by a Georg Forster Research Fellowship from the Alexander von Humboldt Foundation and H.-J. Schmidt for invitation to Potsdam Universität für research.

[1] A. Salam and J. Strathdee, Ann.Phys. (NY) 141, 316 (1982); R. Percacci, J.Math.Phys. 24, 807 (1983).

[2] Dzhunushaliev, V. D. (1993). Izv. Vuzov, ser.Fizika, No. 6, 78 (in Russian); Grav. Cosmol., 3, 240(1998); Gen. Relat. Grav., 30, 583(1998).

[3] T. Levi-Civita, Rend. Atti Acad. Naz. Lincei, 26, 529 (1917).

[4] V.D.Dzhunushaliev and D.Singleton, Phys. Rev. D59, 0664018, (1999) (gr-qc/9807086)

[5] A. Chodos and S. Detweiler, Gen . Rel. Grav., 14, 879 (1982)

[6] M.J. Perry, Phys. Lett. B137, 171 (1984)

[7] J. Maldacena, Adv. Theor. Math. Phys. 2, 231 (1998) (hep-th/9711200)

[8] V.Dzhunushaliev, Mod. Phys. Lett.A, 13, 2179(1998) (gr-qc/9807080)

[9] V.D.Dzhunushaliev, "The model of the piecewise compactification", to be published in the Proceedings of conference "Physical Interpretation of Relativity Theory", London, 11-14 September, 1998.

[10] V. Dzhunushaliev and D. Singleton, Class. Quant. Grav., 16, 973 (1999) gr-qc/9905104)

[11] S.F. Ross, Phys. Rev. D49, 6599 (1994)

[12] S.W. Hawking and S.F. Ross, Phys. Rev. D52, 5865, 1995 
[13] N. Arkani-Hamed, S. Dimopoulos, and G, Dvali, Phys. Lett. B429 263 (1998)

[14] A Davidson and D. Karasik, gr-qc/9901002) 